happiness of individuals to affect the well-being of entire communities.

Results Our strategies are addressed to prevention (al all levels). We deal with violence taking steps to understand the range of factors that put people at risk for violence and protect them from experiencing or perpetrating violence. Our fundamental goal is to stop IPV before it begins. Networks "Violence's Group: Information, Research, Intervention" (2002), "School Against Violence" (2007), along with the training of 480 professionals (health, other sectors) in our region (2009-2012), in the area of prevention of IPV, are examples of our networking strategy. The Family Violence Unit, UVF (2004) - CHUC, integrates both networks; of the 1400 cases (2/3 women, $1 / 3$ men) at UVF, we did not record any murder to date. Our strategy is recognised at regional and national levels.

Conclusions We believe that prevent efforts associated to investigation and a networking strategy (from micro to macrosystem, throughout the life cycle), at individual, family, community, and political levels, are one of the keys to prevent IPV.

\section{THE ONLINE SHELTER - WEB SERVICE FOR EVERYONE CONCERNED OF DOMESTIC VIOLENCE}

Tuulia Kovanen. the Federation of Mother and Child Homes and Shelters, Finland

\subsection{6/injuryprev-2016-042156.989}

Background The Federation of Mother and Child Homes and Shelters is the national child welfare organisation with 30 member associations around Finland. The aim of the Federation is to support parenthood and families, to prevent violence in families and to safeguard the child's right to safe development and childhood. The Online Shelter is a web service maintained by the Federation.

Description of the problem The Online Shelter was founded on 2001 and there are about 5000 visitors monthly. The Online Shelter is a web service for everyone concerned of domestic violence. The aim is that everyone has an equal possibility to get help and support for domestic violence. For example living in a small town can limit your possibility to get help. There is also lot of emotions, like shame and quilt, which are related to domestic violence and can affect your possibility to get help. When it comes to children and youth, internet is the place where they search for help. In these cases the online help can be the only possibility to find and get help.

Results The main aim of the Online Shelter is to offer preventative information about domestic violence and to strengthen people to search for help as early as possible. The Online Shelter provides different kind of help services and tools for surviving. The online chat is usually the first place where people tell about the violence in their lives. Safety is the most important thing in the discussions and it is important that people get the feeling that the chat raises their safety and encourages them to search help from the real time services.

Conclusions This presentation introduces the Online Shelters possibilities to help and give information about domestic violence. This presentation also gives some ideas how to work online, what are the main questions in the Online Shelters chats and what is the meaning of the online help.

\section{PREDICTORS FOR PSYCHOLOGICAL DATING VIOLENCE VICTIMISATION AND PERPETRATION IN MALE AND FEMALE ADOLESCENTS}

${ }^{1}$ Dimitrinka Jordanova Peshevska, ${ }^{2}$ Nikolina Kenig, ${ }^{3}$ Jordanova Tamara. ${ }^{1}$ Psychologist, WHO Consultant on Violence Prevention, Skopje; ${ }^{2}$ Assistance Professor, Instutute of Psychology, Faculty of Philosophy in Skopje; ${ }^{3}$ MSc Candidate, University of Sheffield, Thessaloniki

\subsection{6/injuryprev-2016-042156.990}

Background Adolescent dating violence is an increasing public health problem, viewed in line with the ecological model of influence of different factor. Studies have found that $16 \%$ to $90 \%$ of adolescents have experienced some form of violence from a dating partner. The study objective was to examine the predictors for higher level of psychological dating violence victimisation and perpetration groups in male and female adolescents, such as: with substance use, self-esteem, hostility, conflict resolution skills, gender stereotypes and acceptance of violence.

Methods The convenient sample of 410 students from general/ vocational high schools responded to a self-admistrated 7 scales questionnaire. Informed consent and parent consent were provided for adolescents below 18 years prior. The two groups were divided according to the level of prevalence of psychological victimisation and perpetration into mild and higher. The Cronbach's Alpha of the scales varied from 0.72 to 0.80 . Statistical analysis involved descriptive statistics and logistic regression.

Results A logistic regression analysis performed predicted that: lower self-esteem $(r=-0.077, p<0.05)$, hostility $(r=0.028$, $\mathrm{p}<0.05)$, and acceptance of violence $(r=0.098, p<0.05)$ increase one time more the likelihood for psychological dating violence victimisation in male adolescents. Psychological dating violence victimisation in female adolescents was significantly predicted by: substance use $(r=0.161, p<0.05)$ and acceptance of violence $(r=0.226, p<0.01)$. Psychological dating violence victimisation in male adolescents is significantly distinguished by the predictors such as: substance use $(r=0.188, p<0.05)$ and acceptance of violence $(r=0.286, p<0.01)$, while in female adolescents is the acceptance of violence $(r=0.349, p<0.01)$, increasing the likelihood for 1.4 for times more for psychological dating violence victimisation.

Conclusions Targeting various risk factors should anticipate analysis while designing violence preventive programmes and intervention targeting adolescents.

\section{ARE ALCOHOL ABUSE AND MENTAL HEALTH PROBLEMS ASSOCIATED WITH EXPERIENCE OF INTIMATE PARTNER VIOLENCE AMONG PERSON WITH CHILDREN}

${ }^{1,2}$ Marianne Sipilä, ${ }^{1,2}$ Tuovi Hakulinen, ${ }^{2}$ Eija Paavilainen. ${ }^{1}$ National Institute for Health and Welfare, Finland; ${ }^{2}$ University of Tampere, Finland

\subsection{6/injuryprev-2016-042156.991}

Background Intimate partner violence is a serious problem worldwide and has long been identified as a significant health endangering factor. This study aimed to investigate the associations of intimate partner violence with alcohol abuse and mental health problems among persons with children.

Methods We used population-based cross-sectional data from self-administered questionnaires collected in 2012-2013. The 
sample was randomly selected from the National Population Register in Finland for the Regional Health and Well-being Survey $(\mathrm{ATH})(\mathrm{N}=65$ 000, response rate 53\%). Respondents with children under the age of 18 were selected to this study $(n=6290)$. Associations between intimate partner violence and socio-economic factors, depression, alcohol use, and suicidal intention were analysed using logistic regression.

Results Among those living with children $10.8 \%$ of men and $11.2 \%$ of women reported experiences of intimate partner violence. Experiences of violence were most prevalent in the age group of less than forty years, among those who were married or cohabited (81\%) compared to single parent, and who had basic education or secondary vocational level training compared to polytechnic or higher level training. In the univariate analysis factors which were statistically significantly associated with the experience of violence were cohabited (OR 1.0), having suicidal intentions $(\mathrm{OR}=3.24)$ and reporting melancholic feelings or depression during last 12 months $(\mathrm{OR}=2.48)$. Those with polytechnic or higher level education had slightly smaller odds for experiencing violence than those having basic education or less secondary level education. Alcohol consumption was not associated with experience of violence in this sample.

Conclusions This national survey shows the necessity to recognise intimate partner violence and its associations to alcohol use, low mood, depression and suicidal intentions. These associations should be addressed when developing health and social services and health promotion.

\section{Occupational Safety}

\section{Post Wed 3.8}

\section{TIME TRENDS IN WORK-RELATED INJURIES IN QATAR: AN ANALYSIS OF HOSPITAL TRAUMA REGISTRY DATA}

${ }^{1}$ Amber Mehmood, ${ }^{2}$ Rafael Consunii, ${ }^{2}$ Ruben Peralta, ${ }^{2}$ Rania Abdelhamid, ${ }^{2}$ Ayman ElMenyar, ${ }^{1}$ Katharine A Allen, ${ }^{2}$ Shahnaz Malik, ${ }^{2}$ Sam Thomas, ${ }^{2}$ Hassan Al-Thani, ${ }^{1}$ Adnan Hyder. ${ }^{1} J o h n s$ Hopkins Bloomberg School of Public Health, U.S.A; ${ }^{2}$ Hamad Medical Corporation, Qatar

\subsection{6/injuryprev-2016-042156.992}

Background Work-related injuries (WRIs) are a leading cause of hospital visits in Qatar. Little is known about the burden of hospital admissions due to work related injuries. This study analysed hospital based trauma registry data for past seven years to understand the burden and outcome of these patients.

Methods A retrospective analysis of data from 2008-2014 was conducted on patients admitted to the Hamad Medical Corporation (HMC) Trauma Centre, the national trauma referral centre, and its mortuary with severe WRIs. These were defined as those suffered during working hours or while travelling to or from work and severe enough to need admission to the hospital; poisoning or heat related illnesses were excluded. Temporal trends in the burden, external causes of injuries and mortality rates were analysed.

Results A total of 3,980 severe WRIs were recorded during the study period. Most workers were males (93.4\%) with a mean age of 29.3 years. Almost all workers (98\%) were expatriates. Occupation was known for only $37.5 \%$ of cases, and out of those $80 \%$ were general labourers. Over $39 \%$ of all WRIs were transport injuries. Other important causes were falls (25\%) and exposure to inanimate mechanical forces (14\%). During the study period, severe WRI rates declined from 50.24 to 31.64 per 100,000 registered workers. Overall in-hospital mortality was $12.9 \%$, with a decline from $15.5 \%$ to $9.6 \%$ between 2008 and 2014. The most common cause of WRI fatality was falls (49.5\%).

Conclusions Severe WRI incidence and in-hospital mortality rates have been reduced by almost $40 \%$ in the past 7 years in Qatar. However, we need to also document the burden and outcomes of less severe WRIs and those seen and treated by other providers. Further studies are needed to determine the true burden of workrelated injuries in Qatar, with a focus on unifying national WRI data in order to better inform strategies for occupational safety and injury prevention. Road safety for workers must be a priority in Qatar.

\section{NURSES WELLBEING - EXPOSING FACTOR TO VIOLENT ASSAULTS BY PATIENTS}

${ }^{1}$ Virve Pekurinen, ${ }^{1}$ Minna Anttila, ${ }^{1,2}$ Maritta Välimäki. ${ }^{1}$ Department of Nursing Science, University of Turku, Finland; ${ }^{2}$ Hospital District of Southwest Finland, Finland

\subsection{6/injuryprev-2016-042156.993}

Background Over 64\% of nurses are exposed to violent assaults by patients worldwide. This creates costs for organisations and effect on nurses' health. To prevent assaults, the causes should be known. It has been suggested, that patient characteristics explain only a proportion of assaults, while nurses' wellbeing related factors might contribute to assaults. However, little is known about the specific wellbeing related factors exposing nurses to assaults. The objective of the abstract is to describe these factors exposing nurses to violent assaults by patients in healthcare.

Methods Review of the literature with search terms violence, assaults, aggression, nurse and patient was conducted. Databases used were Cinahl, Pubmed, PsychInfo and Cochrane (20042015). Also manual searches were used. Studies were excluded if: they were conducted in other setting than healthcare, perpetrators were not patients or something else than quantitative research design was used. Searches resulted in 2368 citations, from which 14 studies met the inclusion criteria.

Results Nurses' anxiety, depression, fatigue, psychological distress, burn-out, stress and decreased psychological quality of life seem to expose them to assaults. Also nurses' experiences of increased job demands, poor job control, job strain, time pressure, physical strain and excessive amount of work seem to be their exposing factor to violent assaults by patients. Further, nurses' feelings of fear and guilt, as well as lowered job satisfaction might expose them to assaults.

Conclusions Several wellbeing-related factors seem to expose nurses' to violent assaults realised by patients. These are related to both psychological health and working conditions. Nurses' psychological health and working conditions should be evaluated, monitored and supported as to reduce assaults towards them by patients. Longitudinal research is also needed to verify the results due to mainly cross-sectional nature of the studies.

\section{THE FINNISH TRANSPORT AGENCY'S AND INFRA CONTRACTORS ASSOCIATION'S JOINT VENTURES IN FINLAND}

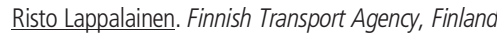

10.1136/injuryprev-2016-042156.994 